\title{
Geometry of non-supersymmetric three-charge bound states*
}

\author{
Eric G. Gimon, ${ }^{1}$ Thomas S. Levi, ${ }^{2}$ and Simon F. Ross ${ }^{3}$ \\ ${ }^{1}$ Berkeley Center for Theoretical Physics, University of California \\ and Lawrence Berkeley National Laboratory, Berkeley, CA 94720, U.S.A. \\ ${ }^{2}$ Center for Cosmology and Particle Physics \\ New York University, 4 Washington Place, New York, NY 10003, U.S.A. \\ ${ }^{3}$ Centre for Particle Theory, Department of Mathematical Sciences \\ University of Durham, South Road, Durham DH1 3LE, U.K.
}

May 2007

\footnotetext{
*This work was supported in part by the Director, Office of Science, Office of High Energy Physics, of the U.S. Department of Energy under Contract No. DE-AC02-05CH11231.
}

\author{
DISCLAIMER
}

This document was prepared as an account of work sponsored by the United States Government. While this document is believed to contain correct information, neither the United States Government nor any agency thereof, nor The Regents of the University of California, nor any of their employees, makes any warranty, express or implied, or assumes any legal responsibility for the accuracy, completeness, or usefulness of any information, apparatus, product, or process disclosed, or represents that its use would not infringe privately owned rights. Reference herein to any specific commercial product, process, or service by its trade name, trademark, manufacturer, or otherwise, does not necessarily constitute or imply its endorsement, recommendation, or favoring by the United States Government or any agency thereof, or The Regents of the University of California. The views and opinions of authors expressed herein do not necessarily state or reflect those of the United States Government or any agency thereof or The Regents of the University of California. 
DCPT-07/19

\title{
Geometry of non-supersymmetric three-charge bound states
}

\author{
Eric G. Gimon ${ }^{\Uparrow}$, Thomas S. Levi狍 and Simon F. Ross \\ ${ }^{a}$ Berkeley Center for Theoretical Physics, Berkeley, CA 94720, U.S.A. \\ ${ }^{b}$ Center for Cosmology and Particle Physics \\ New York University, 4 Washington Place, New York, NY 10003, U.S.A. \\ ${ }^{c}$ Centre for Particle Theory, Department of Mathematical Sciences \\ University of Durham, South Road, Durham DH1 3LE, U.K.
}

\begin{abstract}
We study the smooth non-supersymmetric three-charge microstates of Jejjala, Madden, Ross and Titchener [1] using Kaluza-Klein reductions of the solutions to five and four dimensions. Our aim is to improve our understanding of the relation between these non-supersymmetric solutions and the well-studied supersymmetric cases. We find some surprising qualitative differences. In the five-dimensional description, the solution has orbifold fixed points which break supersymmetry locally, so the geometries cannot be thought of as made up of separate half-BPS centers. In the four-dimensional description, the two singularities in the geometry are connected by a conical singularity, which makes it impossible to treat them independently and assign unambiguous brane charges to these centers.
\end{abstract}

\section{Introduction}

The study of black holes and their thermodynamics has led to a number of important advances in string theory. Recently, the construction of smooth geometries corresponding to individual microstates of the black holes (more precisely, in a dual field theory description, to individual pure states which contribute to the thermal ensemble) has received extensive attention. Supersymmetric two-charge microstates are now reasonably well-understood [2, 3, 4, 5, 6, 7, 8, 9, 10] (see [11, 12] for a review), and substantial progress has been made on understanding the supersymmetric threecharge microstates $[13,14,15,16,17,18,19,20,21]$ and the dimensional reduction to

*eggimon@lbl.gov

${ }^{\dagger}$ levi@physics.nyu.edu

‡S.F.Ross@durham.ac.uk 
four dimensions $[22,23,24,25]$. Our aim in this paper is to continue the investigation of non-supersymmetric states initiated in [1].

In the three-charge case, an important step was the rewriting [16] of the first examples of smooth three-charge geometries in the fibered form used in the classification of supersymmetric solutions [26]. This led to the realisation that the base space for these solutions had a "pseudo-hyper-Kähler" form, which led to the generalisations to multi-center solutions [17, 18]. These solutions all have a $U(1)$ isometry in the base. This may appear to be an unnatural restriction for the five-dimensional case, but if we add a NUT charge and reduce along the isometry direction, this family of solutions naturally corresponds to microstates of four-dimensional asymptotically flat black holes [22, 23, 24]. This focus on the centers was refined in [24], where it was conjectured that the general four-dimensional supersymmetric microstate is made up of half-BPS "atoms". This will be described by a multi-center geometry if the distance between the individual "atoms" is sufficiently large, or by passing to an open string description if the separations are small. This separation is modulated by the string coupling.

We would like to apply the lessons learnt from this analysis to the non-supersymmetric solutions of [1]. These solutions are qualitatively similar to the simplest supersymmetric two-center three-charge solutions studied in [14, 15, 16, so analysing them in a similar way should shed some light on the similarities and differences, and ultimately guide us towards constructing more general non-supersymmetric microstates. The plan is thus to first Kaluza-Klein reduce the solutions to five dimensions, where we can see that the solutions have two centers. We will give a description of the five-dimensional solution as a $T^{6}$ compactification from M-theory, which makes the symmetry between the charges manifest. This five-dimensional solution has a $U(1)$ isometry in the base. It is natural to consider a further dimensional reduction to four dimensions along this isometry to attempt to obtain a more direct physical interpretation, as in [24].

Implementing this program leads to some surprises: first, in the five-dimensional solution, the two centers are locally described by non-supersymmetric orbifolds. We had expected to find an orbifold singularity at these points in the five dimensional description, but it is a surprise that the supersymmetry is broken even locally: in the sixdimensional description, the full asymptotically flat solution is non-supersymmetric, but it has (for suitable choices of parameters) a near-core $\mathrm{AdS}_{3} \times S^{3}$ geometry, in which supersymmetry is restored. This supersymmetry is broken by the choice of Kaluza-Klein reduction: even if we consider the Kaluza-Klein reduction from the exact $\mathrm{AdS}_{3} \times S^{3}$ geometry, the five-dimensional solution contains non-supersymmetric orbifold singularities. This leads to the first general lesson we wish to draw: the picture of microstates as made up of half-BPS "atoms" does not extend to the nonsupersymmetric case. We must consider more general kinds of basic building blocks.

If we reduce to four dimensions, we find our second surprise: the four-dimensional solution is not smooth away from the centers. There is a conical singularity which connects the two centers. The ambiguity in the definition of the Kaluza-Klein gauge field on this conical line singularity makes it impossible for us to unambiguously associate brane charges with the two centers. This suggests that unlike in the supersymmetric 
case, we cannot treat the two centers independently, and the physical interpretation of these centers in the four-dimensional description is much less clear here.

One of the ultimate goals of this work was to make progress towards constructing more general non-supersymmetric solutions. In general, constructing non-supersymmetric solutions is much more difficult than supersymmetric ones. In the nonsupersymmetric case we have to deal directly with the non-linear, second order equations of motion, which makes it impossible to construct solutions in terms of harmonic functions, as can be done in the supersymmetric case. The results we have obtained show that the non-supersymmetric case is quite different from the supersymmetric one, and suggest that it will be difficult to extend the work on supersymmetric cases to construct multi-center non-supersymmetric solutions. However, it may still be possible at least to construct non-supersymmetric solutions which are asymptotically flat in four dimensions; work on this problem is ongoing [27].

The plan of the rest of the paper is as follows: in the next section, we review the non-supersymmetric solitons of [1] in type IIB supergravity. In section 3, we convert these solutions to an M-theory form, write the metric in terms of the integer parameters of the solitons, and discuss the relation to the supersymmetric case. In section 4, we analyse the structure of the M-theory solutions, with a particular focus on the orbifold singularities appearing in this five-dimensional description. Finally, in section 5, we discuss the dimensional reduction to four dimensions.

\section{Review of construction}

We start from the metric for the D1-D5-P black string, written in the fibered form in [1],

$$
\begin{aligned}
\mathrm{d} s^{2} & =\frac{1}{\sqrt{\tilde{H}_{1} \tilde{H}_{5}}}\left\{-(f-M)\left[\mathrm{d} \tilde{t}-(f-M)^{-1} M \cosh \delta_{1} \cosh \delta_{5}\left(a_{1} \cos ^{2} \bar{\theta} \mathrm{d} \psi+a_{2} \sin ^{2} \bar{\theta} \mathrm{d} \phi\right)\right]^{2}\right. \\
& \left.+f\left[\mathrm{~d} \tilde{y}+f^{-1} M \sinh \delta_{1} \sinh \delta_{5}\left(a_{2} \cos ^{2} \bar{\theta} \mathrm{d} \psi+a_{1} \sin ^{2} \bar{\theta} \mathrm{d} \phi\right)\right]^{2}\right\} \\
& +\sqrt{\tilde{H}_{1} \tilde{H}_{5}}\left\{\frac{\bar{r}^{2} \mathrm{~d} \bar{r}^{2}}{\left(\bar{r}^{2}+a_{1}^{2}\right)\left(\bar{r}^{2}+a_{2}^{2}\right)-M \bar{r}^{2}}+\mathrm{d} \bar{\theta}^{2}\right. \\
& +(f(f-M))^{-1}\left[\left(f(f-M)+f a_{2}^{2} \sin ^{2} \bar{\theta}-(f-M) a_{1}^{2} \sin ^{2} \bar{\theta}\right) \sin ^{2} \bar{\theta} \mathrm{d} \phi^{2}\right. \\
& +2 M a_{1} a_{2} \sin ^{2} \bar{\theta} \cos ^{2} \bar{\theta} \mathrm{d} \psi \mathrm{d} \phi \\
& \left.\left.+\left(f(f-M)+f a_{1}^{2} \cos ^{2} \bar{\theta}-(f-M) a_{2}^{2} \cos ^{2} \bar{\theta}\right) \cos ^{2} \bar{\theta} \mathrm{d} \psi^{2}\right]\right\}+\sqrt{\frac{\tilde{H}_{1}}{\tilde{H}_{5}}} \sum_{i=1}^{4} d z_{i}^{2},
\end{aligned}
$$

where $\tilde{t}=t \cosh \delta_{p}-y \sinh \delta_{p}, \tilde{y}=y \cosh \delta_{p}-t \sinh \delta_{p}$,

$$
\tilde{H}_{i}=f+M \sinh ^{2} \delta_{i}, \quad f=\bar{r}^{2}+a_{1}^{2} \sin ^{2} \bar{\theta}+a_{2}^{2} \cos ^{2} \bar{\theta} .
$$

This metric is more usually written in terms of functions $H_{i}=\tilde{H}_{i} / f$. Writing it in this way instead makes it clear that there is no singularity at $f=0$. The above 
metric is in the string frame, and the dilaton is

$$
e^{2 \Phi}=\frac{\tilde{H}_{1}}{\tilde{H}_{5}}
$$

The 2-form gauge potential which supports this configuration is

$$
\begin{aligned}
C_{2} & =\frac{M \cos ^{2} \bar{\theta}}{\tilde{H}_{1}}\left[\left(a_{2} c_{1} s_{5} c_{p}-a_{1} s_{1} c_{5} s_{p}\right) d t+\left(a_{1} s_{1} c_{5} c_{p}-a_{2} c_{1} s_{5} s_{p}\right) d y\right] \wedge d \psi \\
+ & \frac{M \sin ^{2} \bar{\theta}}{\tilde{H}_{1}}\left[\left(a_{1} c_{1} s_{5} c_{p}-a_{2} s_{1} c_{5} s_{p}\right) d t+\left(a_{2} s_{1} c_{5} c_{p}-a_{1} c_{1} s_{5} s_{p}\right) d y\right] \wedge d \phi \\
& -\frac{M s_{1} c_{1}}{\tilde{H}_{1}} d t \wedge d y-\frac{M s_{5} c_{5}}{\tilde{H}_{1}}\left(\bar{r}^{2}+a_{2}^{2}+M s_{1}^{2}\right) \cos ^{2} \bar{\theta} d \psi \wedge d \phi,
\end{aligned}
$$

where $c_{i}=\cosh \delta_{i}, s_{i}=\sinh \delta_{i}$. We take the $T^{4}$ in the $z_{i}$ directions to have volume $V$, and the $y$ circle to have radius $R$, that is $y \sim y+2 \pi R$.

This metric and fields describes a family of solutions with D1, D5 and P charges, labelled by the seven parameters $M, a_{1}, a_{2}, \delta_{1}, \delta_{2}, \delta_{3}, R$. It was shown in [1] that the geometry is smooth everywhere and contains no closed timelike curves if these parameters are fixed in terms of two integers $m, n$ and the charges $q_{1}, q_{5}, q_{p}$ by the relations

$$
\begin{gathered}
M=a_{1}^{2}+a_{2}^{2}-a_{1} a_{2} \frac{\left(c_{1}^{2} c_{5}^{2} c_{p}^{2}+s_{1}^{2} s_{5}^{2} s_{p}^{2}\right)}{s_{1} c_{1} s_{5} c_{5} s_{p} c_{p}}, \\
\frac{s_{p} c_{p}}{\left(a_{1} c_{1} c_{5} c_{p}-a_{2} s_{1} s_{5} s_{p}\right)} R=n, \quad \frac{s_{p} c_{p}}{\left(a_{2} c_{1} c_{5} c_{p}-a_{1} s_{1} s_{5} s_{p}\right)} R=-m, \\
R=\frac{M s_{1} c_{1} s_{5} c_{5}\left(s_{1} c_{1} s_{5} c_{5} s_{p} c_{p}\right)^{1 / 2}}{\sqrt{a_{1} a_{2}}\left(c_{1}^{2} c_{5}^{2} c_{p}^{2}-s_{1}^{2} s_{5}^{2} s_{p}^{2}\right)} .
\end{gathered}
$$

If we introduce dimensionless parameters

$$
j=\left(\frac{a_{2}}{a_{1}}\right)^{1 / 2} \leq 1, \quad s=\left(\frac{s_{1} s_{5} s_{p}}{c_{1} c_{5} c_{p}}\right)^{1 / 2} \leq 1,
$$

then the integer quantisation conditions determine these via

$$
\frac{j+j^{-1}}{s+s^{-1}}=m-n, \quad \frac{j-j^{-1}}{s-s^{-1}}=m+n
$$

Which gives

$$
s^{2}=\frac{m^{2}+n^{2}-1-\sqrt{\left(m^{2}-n^{2}\right)^{2}-2\left(m^{2}+n^{2}\right)+1}}{2 m n} .
$$

Note that this constraint is invariant under the permutation of the three charges. We can rewrite the mass (2.5) as

$$
M=a_{1} a_{2}\left(s^{2}-j^{2}\right)\left(j^{-2} s^{-2}-1\right)=a_{1} a_{2} n m\left(s^{-2}-s^{2}\right)^{2},
$$


so having solved for $s$ in terms of $m, n$, we can use this to write $a_{1} a_{2}$ in terms of $M$. The only remaining step is to solve for $M$ in terms of $m, n$ and the charges. This was left entirely implicit in [1]; we will say a little more about it below.

An unsatisfactory feature of this story is the highly implicit nature of the above conditions. We will see below that we can write the metric explicitly in terms of the integer quantised parameters $m, n$ and a single length scale, which is related to $M$ and hence determined in terms of the charges $q_{i}$. This will bring the analysis much closer to the supersymmetric cases. It is worth pointing out at this stage that it would equally have been possible to rewrite it in this way in the type IIB form above; this rewriting is independent of the transformation to the M-theory form.

In what follows, we will usually assume $m, n$ are coprime. If we do not, there will be additional orbifold singularities in the M-theory form of the solution.

\section{M-theory form}

The general study of solutions in the supersymmetric case is conducted in an Mtheory picture, where the symmetry between the three charges is manifest. Our first task is therefore to translate the type IIB form in which the non-supersymmetric solutions were first obtained to an M-theory form.

To pass to the M-theory form, we T-dualise on $z_{3}, z_{4}$ to get to a D3-D3-P solution, and then T-dualise on $y$ to get to D2-D2-F1 in IIA. The IIA solution is then uplifted to M-theory. There is a symmetry between the charges in the M-theory picture, so we rename the charges to $Q_{1}=q_{1}, Q_{2}=q_{5}, Q_{3}=q_{p}$. For the general family of rotating black string solutions (2.1), the resulting solution is

$$
d s_{11}^{2}=-\left(\tilde{H}_{1} \tilde{H}_{2} \tilde{H}_{3}\right)^{-2 / 3} f(f-M)(d t+k)^{2}+\left(\tilde{H}_{1} \tilde{H}_{2} \tilde{H}_{3}\right)^{1 / 3} d \bar{s}_{4}^{2}+d s_{T^{6}}^{2},
$$

where

$$
k=-\frac{M c_{1} c_{2} c_{3}}{f-M}\left(a_{1} \cos ^{2} \bar{\theta} d \psi+a_{2} \sin ^{2} \bar{\theta} d \phi\right)+\frac{M s_{1} s_{2} s_{3}}{f}\left(a_{2} \cos ^{2} \bar{\theta} d \psi+a_{1} \sin ^{2} \bar{\theta} d \phi\right),
$$

and the four-dimensional base metric is

$$
\begin{aligned}
\overline{d s}_{4}^{2} & =\frac{\bar{r}^{2} d \bar{r}^{2}}{g(\bar{r})}+d \bar{\theta}^{2} \\
& +(f(f-M))^{-1}\left[\left(f(f-M)+f\left(a_{2}^{2}-a_{1}^{2}\right) \sin ^{2} \bar{\theta}+M a_{1}^{2} \sin ^{2} \bar{\theta}\right) \sin ^{2} \bar{\theta} d \phi^{2}\right. \\
& +2 M a_{1} a_{2} \sin ^{2} \bar{\theta} \cos ^{2} \bar{\theta} d \phi d \psi \\
& \left.+\left(f(f-M)+f\left(a_{1}^{2}-a_{2}^{2}\right) \cos ^{2} \bar{\theta}+M a_{2}^{2} \cos ^{2} \bar{\theta}\right) \cos ^{2} \bar{\theta} d \psi^{2}\right],
\end{aligned}
$$

where

$g(\bar{r})=\left(\bar{r}^{2}+a_{1}^{2}\right)\left(\bar{r}^{2}+a_{2}^{2}\right)-M \bar{r}^{2} \equiv\left(\bar{r}^{2}-r_{+}^{2}\right)\left(\bar{r}^{2}-r_{-}^{2}\right), \quad f=\bar{r}^{2}+a_{1}^{2} \sin ^{2} \bar{\theta}+a_{2}^{2} \cos ^{2} \bar{\theta}$.

Note that the coordinate $\bar{r}$ lives on the interval $\left[r_{+}, \infty\right)$; this fact will become clearer in some of the later coordinate systems. The metric is asymptotically flat in five 
dimensions. The coordinates $\bar{\theta}, \phi, \psi$ are conventional coordinates on the $S^{3}$ at large $\bar{r}$. The metric on the $T^{6}$, which plays little part in our discussions, is

$$
d s_{T^{6}}^{2}=\left(\tilde{H}_{1} \tilde{H}_{2} \tilde{H}_{3}\right)^{1 / 3}\left(\tilde{H}_{1}^{-1}\left(d z_{1}^{2}+d z_{2}^{2}\right)+\tilde{H}_{2}^{-1}\left(d z_{3}^{2}+d z_{4}^{2}\right)+\tilde{H}_{3}^{-1}\left(d z_{5}^{2}+d z_{6}^{2}\right)\right) .
$$

The gauge field in eleven dimensions is

$$
C_{(3)}=A_{1} \wedge d z_{1} \wedge d z_{2}+A_{2} \wedge d z_{3} \wedge d z_{4}+A_{3} \wedge d z_{5} \wedge d z_{6}
$$

where

$$
A_{i}=-\frac{1}{\tilde{H}_{i}}\left[Q_{i} d t+M c_{j} c_{k} s_{i}\left(a_{1} \cos ^{2} \bar{\theta} d \psi+a_{2} \sin ^{2} \bar{\theta} d \phi\right)-M s_{j} s_{k} c_{i}\left(a_{2} \cos ^{2} \bar{\theta} d \psi+a_{1} \sin ^{2} \bar{\theta} d \phi\right)\right]
$$

where here and in similar formulae throughout, $j$ and $k$ denote the other two charge parameters, $i \neq j \neq k$ (so if $i=1, j=2$ and $k=3$, and so forth). The charges are defined as

$$
Q_{i}=M \sinh \delta_{i} \cosh \delta_{i}
$$

\subsection{Adapted coordinates}

The foregoing discussion applies to the general family of solutions (2.1); we would like to specialise to the smooth soliton solutions, and eliminate the dependence on the parameters to rewrite this solution in a way which makes the dependence on the integer parameters $m, n$ manifest. This will be helpful in understanding the relation to the supersymmetric cases, and will also make the structure of our non-supersymmetric solution clearer. In this subsection, we introduce a new coordinate system adapted to this solution. In the next, we will consider coordinates which are as close as possible to the coordinates used in the supersymmetric cases.

We set

$$
\rho^{2}=\frac{\bar{r}^{2}-r_{+}^{2}}{r_{+}^{2}-r_{-}^{2}}, \quad x=\cos 2 \bar{\theta} .
$$

The range of the coordinates is $\rho \geq 0,-1 \leq x \leq 1$. In these coordinates,

$$
\frac{\bar{r}^{2} d \bar{r}^{2}}{g(\bar{r})}=\frac{d \rho^{2}}{\rho^{2}+1} \text {. }
$$

and

$f-\frac{1}{2} M=\left(r_{+}^{2}-r_{-}^{2}\right) \rho^{2}+\frac{1}{2}\left(a_{2}^{2}-a_{1}^{2}\right) x+r_{+}^{2}+\frac{1}{2}\left(a_{1}^{2}+a_{2}^{2}-M\right)=2 c\left(2 \rho^{2}+1-\left(m^{2}-n^{2}\right) x\right)$,

where

$$
c \equiv \frac{r_{+}^{2}-r_{-}^{2}}{4}
$$

Thus,

$V \equiv \frac{f(f-M)}{4 c^{2}}=4 \rho^{2}\left(\rho^{2}+1\right)-\left(m^{2}-n^{2}\right)^{2}\left(1-x^{2}\right)-2\left(m^{2}-n^{2}\right) x\left(2 \rho^{2}+1\right)+2\left(m^{2}+n^{2}\right)$. 
This now involves only the integer parameters $m, n$ and the single length scale $c$. We can continue to calculate the full metric, and we find that we can in a similar fashion eliminate all the dependence on the parameters in favour of $m, n$ and the single length scale $c$ by fully exploiting (2.5.2.7). Some useful relations which follow from (2.5.2.7) are

$$
\begin{aligned}
& M a_{1} a_{2}=16 c^{2} n m, \quad a_{1}^{2}-a_{2}^{2}=4 c\left(m^{2}-n^{2}\right), \\
& \frac{1}{2} M\left(a_{1}^{2}+a_{2}^{2}\right)=8 c^{2}\left[\left(m^{2}-n^{2}\right)^{2}-\left(m^{2}+n^{2}\right)\right], \\
& \frac{1}{4} M^{2}=4 c^{2}\left[\left(m^{2}-n^{2}\right)^{2}-2\left(m^{2}+n^{2}\right)+1\right] .
\end{aligned}
$$

Omitting the details of the calculation, we find

$$
\begin{aligned}
k=\frac{\sqrt{Q_{1} Q_{2} Q_{3}}}{4 c V \sqrt{m n}}\left\{\left[2 \rho^{2}-\left(m^{2}-n^{2}\right)(1+x)\right] n(1-x) d \phi\right. \\
\left.-\left[2 \rho^{2}+\left(m^{2}-n^{2}\right)(1-x)\right] m(1+x) d \psi\right\},
\end{aligned}
$$

and the base metric is

$$
\begin{aligned}
\bar{d} s_{4}^{2}= & \frac{d \rho^{2}}{\rho^{2}+1}+\frac{d x^{2}}{4\left(1-x^{2}\right)}+\frac{2 n m}{V}\left(1-x^{2}\right) d \phi d \psi \\
& +\frac{1}{2 V}\left[4 \rho^{2}\left(\rho^{2}+1\right)-\left(m^{2}-n^{2}\right)\left(2 \rho^{2}+1\right)(1+x)+\left(m^{2}+n^{2}\right)(1+x)\right](1-x) d \phi^{2} \\
& +\frac{1}{2 V}\left[4 \rho^{2}\left(\rho^{2}+1\right)+\left(m^{2}-n^{2}\right)\left(2 \rho^{2}+1\right)(1-x)+\left(m^{2}+n^{2}\right)(1-x)\right](1+x) d \psi^{2} .
\end{aligned}
$$

If we define

$$
\phi=\frac{1}{2}(\varphi-\tau), \quad \psi=\frac{1}{2}(\varphi+\tau),
$$

we can also rewrite (3.18) in a fibered form,

$$
\bar{d} s_{4}^{2}=\frac{\left[4 \rho^{2}\left(\rho^{2}+1\right)+(m-n)^{2}\left(1-x^{2}\right)\right]}{4 V}(d \tau+\delta d \varphi)^{2}+d s_{3}^{2},
$$

where

$$
\delta=\frac{\left[4 \rho^{2}\left(\rho^{2}+1\right) x+\left(m^{2}-n^{2}\right)\left(1-x^{2}\right)\left(2 \rho^{2}+1\right)\right]}{\left[4 \rho^{2}\left(\rho^{2}+1\right)+(m-n)^{2}\left(1-x^{2}\right)\right]}
$$

and

$$
d s_{3}^{2}=\frac{d \rho^{2}}{\rho^{2}+1}+\frac{d x^{2}}{4\left(1-x^{2}\right)}+\frac{\left(1-x^{2}\right) \rho^{2}\left(\rho^{2}+1\right)}{\left[4 \rho^{2}\left(\rho^{2}+1\right)+(m-n)^{2}\left(1-x^{2}\right)\right]} d \varphi^{2} .
$$

This fibered form is useful for comparison to the base metric in the supersymmetric case, which is conventionally written in a similar fibered form, and also for analysing the dimensional reduction to four dimensions. The coordinates $\phi$ and $\psi$ are $2 \pi$ periodic. This implies that $\tau$ and $\varphi$ have periodicities

$$
(\tau, \varphi) \sim(\tau-2 \pi, \varphi+2 \pi), \quad \tau \sim \tau+4 \pi
$$


The fermions will be antiperiodic under $(\tau, \varphi) \sim(\tau-2 \pi, \varphi+2 \pi)$ and periodic under $\tau \sim \tau+4 \pi$. The functions $\tilde{H}_{i}$ appearing in the metric become

$$
\tilde{H}_{i}=2 c\left[2 \rho^{2}+1-\left(m^{2}-n^{2}\right) x+E_{i}\right],
$$

where we introduce the convenient constants

$$
E_{i} \equiv \sqrt{\frac{Q_{i}^{2}}{4 c^{2}}+\left[\left(m^{2}-n^{2}\right)^{2}-2\left(m^{2}+n^{2}\right)+1\right]} .
$$

It was shown in [1] that $\tilde{H}_{i}>0$ everywhere which implies $E_{i}>\left(m^{2}-n^{2}\right)-1$. The gauge fields are

$$
\begin{aligned}
A_{i}= & \tilde{H}_{i}^{-1}\left\{Q_{i} d t-\sqrt{\frac{Q_{j} Q_{k}}{Q_{i}}} \frac{c}{\sqrt{m n}}\left[n\left(m^{2}-n^{2}+1+E_{i}\right)(1-x) d \phi\right.\right. \\
& \left.\left.+m\left(m^{2}-n^{2}-1-E_{i}\right)(1+x) d \psi\right]\right\} .
\end{aligned}
$$

We can also write the ADM mass and angular momenta of the five-dimensional asymptotically flat solution in the Einstein frame (in units where $4 G^{(5)} / \pi=1$ ) in terms of the integer parameters as

$$
\begin{gathered}
M_{A D M}=2 c\left(E_{1}+E_{2}+E_{3}\right) \\
J_{\psi}=-\sqrt{\frac{m}{n}} \sqrt{Q_{1} Q_{2} Q_{3}}, \quad J_{\phi}=\sqrt{\frac{n}{m}} \sqrt{Q_{1} Q_{2} Q_{3}} .
\end{gathered}
$$

This coordinate transformation provides us with a nice form of the metric, which makes the special nature of the soliton solutions evident. We see that the base metric is completely independent of the charges or any length scale. The full metric is written in terms of $m, n$, the length scale $c$, and the charges $Q_{i}$, which enter explicitly only through the functions $\tilde{H}_{i}$. However, $c$ is not an independent length scale: it should be determined in terms of the charges and $m, n$. In the supersymmetric case $m=n+1$, we had [16]

$$
c_{\text {susy }}=\frac{1}{4 n(n+1)} \frac{Q_{1} Q_{2} Q_{3}}{Q_{1} Q_{2}+Q_{1} Q_{3}+Q_{2} Q_{3}} .
$$

We should determine how $c$ is related to the charges in the non-supersymmetric case. We first note that (3.12) is equivalent to

$$
c=\frac{M}{4 m n}\left(s^{-2}-s^{2}\right)^{-1} .
$$

Since $s^{2}$ is determined in terms of $m, n$ by (2.10), the problem is simply to determine $M$ in terms of the charges $Q_{i}$ and $m, n$. Using

$$
Q_{i}=M \sinh \delta_{i} \cosh \delta_{i}=M \frac{\tanh \delta_{i}}{1-\tanh ^{2} \delta_{i}}
$$


we can express $s^{2}=\tanh \delta_{1} \tanh \delta_{2} \tanh \delta_{3}$ in terms of the $Q_{i}$ and $M$. We want to solve this for $M$ as a function of $s^{2}$ and the charges. The equation can be rearranged to find that the combination $\bar{M}=M\left(s^{-2}-s^{2}\right)^{-1}=4 m n c$ satisfies

$$
\begin{aligned}
& \left(s^{-2}-s^{2}\right) Q_{1} Q_{2} Q_{3} \bar{M}^{3}\left(Q_{1} \bar{M}+Q_{2} Q_{3}\right)\left(Q_{2} \bar{M}+Q_{1} Q_{3}\right)\left(Q_{3} \bar{M}+Q_{1} Q_{2}\right) \\
& +\left[2 Q_{1}^{2} Q_{2}^{2} Q_{3}^{2}\left(Q_{1}^{2}+Q_{2}^{2}+Q_{3}^{2}\right)-\left(Q_{1}^{4} Q_{3}^{4}+Q_{1}^{4} Q_{2}^{4}+Q_{2}^{4} Q_{3}^{4}\right)\right] \bar{M}^{4}+8 Q_{1}^{3} Q_{2}^{3} Q_{3}^{3} \bar{M}^{3} \\
& +2 Q_{1}^{2} Q_{2}^{2} Q_{3}^{2}\left(Q_{1}^{2} Q_{2}^{2}+Q_{1}^{2} Q_{3}^{2}+Q_{2}^{2} Q_{3}^{2}\right) \bar{M}^{2}-Q_{1}^{4} Q_{2}^{4} Q_{3}^{4}=0 .
\end{aligned}
$$

In the supersymmetric case, $s=1$, the first line is absent, so one can see that (3.29) satisfies this equation. 1 In the non-supersymmetric case, there is no explicit solution for $c$ in general, but it is determined implicitly in terms of the charges by (3.32). Explicit solutions are possible in special cases: If all three charges are equal, we can write

$$
c=\frac{Q}{4 m n} \frac{s^{-2 / 3}-s^{2 / 3}}{s^{-2}-s^{2}} .
$$

If one of the charges is much smaller than the other two, say $Q_{3} \ll Q_{1}, Q_{2}$, then

$$
c \approx \frac{Q_{3}}{4 m n}
$$

This limit is interesting because it is the regime where the type IIB solution has a near-core $\mathrm{AdS}_{3} \times S^{3}$ geometry.

\subsection{Alternative coordinates}

The coordinates above provide the simplest description of the solution, but they are not directly related to the coordinates used for the supersymmetric solutions in [16]. It is therefore useful to introduce an alternative coordinate system which makes the connection to the supersymmetric case clearer. These coordinates will also be adapted to studying the local structure near one of the orbifold singularities, as we will see later.

We therefore introduce new coordinates $r, \theta$ through

$$
\begin{gathered}
r=\frac{\left(\bar{r}^{2}-r_{+}^{2}\right)}{4}+c \sin ^{2} \bar{\theta}=c\left[\rho^{2}+\frac{1}{2}(1-x)\right], \\
r \cos ^{2} \frac{\theta}{2}=\frac{\left(\bar{r}^{2}-r_{+}^{2}\right)}{4} \cos ^{2} \bar{\theta}=\frac{c}{2} \rho^{2}(1+x) .
\end{gathered}
$$

The range of the coordinates is $r \geq 0,0 \leq \theta \leq \pi$. One could similarly define coordinates centered on the singularity at $\rho=0, x=-1$ by

$$
\begin{gathered}
r_{c}=c\left[\rho^{2}+\frac{1}{2}(1+x)\right], \\
r_{c} \cos ^{2} \frac{\theta_{c}}{2}=\frac{c}{2} \rho^{2}(1-x),
\end{gathered}
$$

\footnotetext{
${ }^{1}$ The equation becomes $\left[\left(Q_{1} Q_{2}+Q_{1} Q_{3}+Q_{2} Q_{3}\right) \bar{M}-Q_{1} Q_{2} Q_{3}\right]\left[\left(Q_{1} Q_{2}-Q_{1} Q_{3}-Q_{2} Q_{3}\right) \bar{M}-\right.$ $\left.\left.Q_{1} Q_{2} Q_{3}\right]\left[\left(Q_{1} Q_{2}-Q_{1} Q_{3}+Q_{2} Q_{3}\right) \bar{M}+Q_{1} Q_{2} Q_{3}\right)\right]\left[\left(Q_{1} Q_{2}+Q_{1} Q_{3}-Q_{2} Q_{3}\right) \bar{M}+Q_{1} Q_{2} Q_{3}\right]=0$.
} 
but we will focus henceforth on the $r, \theta$ coordinates. It is useful to retain $r_{c}$, however, thought of as a function of $r, \theta$ given by

$$
r_{c}^{2}=r^{2}+2 r c \cos \theta+c^{2}
$$

then the inverse coordinate transformation is

$$
2 c \sin ^{2} \bar{\theta}=\left(r-r_{c}\right)+c, \quad \bar{r}^{2}-r_{+}^{2}=2\left(r+r_{c}-c\right),
$$

or

$$
\rho^{2}=\frac{\left(r+r_{c}-c\right)}{2 c}, \quad x=\frac{\left(r_{c}-r\right)}{c} .
$$

These coordinates have some nice properties even for the general metric (3.3):

$$
g(\bar{r})=4\left(r+r_{c}\right)^{2}-4 c^{2}
$$

which can be used to show

$$
g(\bar{r}) \sin ^{2} \bar{\theta} \cos ^{2} \bar{\theta}=4 r^{2} \sin ^{2} \theta
$$

and

$$
\frac{\bar{r}^{2} d \bar{r}^{2}}{g(\bar{r})}+d \bar{\theta}^{2}=\frac{1}{4 r r_{c}}\left(d r^{2}+r^{2} d \theta^{2}\right),
$$

so this coordinate transformation casts this part of the metric in a conformally flat form (note that we get a conformally flat form even before restricting to the smooth soliton solutions). The base metric used in the supersymmetric solutions differs from the one used up to now by a conformal factor; define

$$
d s_{4}^{2}=2 c \sqrt{V} \bar{d} s_{4}^{2}
$$

The eleven-dimensional metric is then

$$
d s_{11}^{2}=-\left(Z_{1} Z_{2} Z_{3}\right)^{-2 / 3}(d t+k)^{2}+\left(Z_{1} Z_{2} Z_{3}\right)^{1 / 3} d s_{4}^{2}+d s_{T^{6}}^{2},
$$

with

$$
\begin{gathered}
Z_{i} \equiv \frac{\tilde{H}_{i}}{2 c \sqrt{V}}=\frac{1}{c \sqrt{V}}\left[r+r_{c}+\left(m^{2}-n^{2}\right)\left(r-r_{c}\right)+c E_{i}\right] \\
V=\frac{1}{c^{2}}\left[\left(m^{2}-n^{2}\right)\left(r-r_{c}\right)+\left(r+r_{c}\right)\right]^{2}-\left[\left(m^{2}-n^{2}\right)^{2}-2\left(m^{2}+n^{2}\right)+1\right],
\end{gathered}
$$

and

$$
\begin{aligned}
k= & \frac{\sqrt{Q_{1} Q_{2} Q_{3}}}{8 c^{3} \sqrt{m n} V}\left\{-m\left[\left(m^{2}-n^{2}\right)\left(r-r_{c}+c\right)+\left(r+r_{c}-c\right)\right]\left(r_{c}-r+c\right)(d \tau+d \varphi)\right. \\
& \left.+n\left[\left(m^{2}-n^{2}\right)\left(r-r_{c}-c\right)+\left(r+r_{c}-c\right)\right]\left(r-r_{c}+c\right)(-d \tau+d \varphi)\right\} .
\end{aligned}
$$

The base metric here is (using the angular coordinates (3.19) appropriate for writing the base as a fibration)

$$
d s_{4}^{2}=H^{-1} \sigma^{2}+H\left[\gamma\left(d r^{2}+r^{2} d \theta^{2}\right)+r^{2} \sin ^{2} \theta d \varphi^{2}\right]
$$


with

$$
\begin{aligned}
& H=\frac{2 c \sqrt{V}}{\left[\left(\left(r+r_{c}\right)^{2}-c^{2}\right)-(m-n)^{2}\left(\left(r-r_{c}\right)^{2}-c^{2}\right)\right]}, \\
& \gamma=\frac{\left[\left(\left(r+r_{c}\right)^{2}-c^{2}\right)-(m-n)^{2}\left(\left(r-r_{c}\right)^{2}-c^{2}\right)\right]}{4 r r_{c}},
\end{aligned}
$$

and

$$
\sigma=d \tau-\frac{\left[\left(\left(r+r_{c}\right)^{2}-c^{2}\right)\left(r-r_{c}\right)+\left(\left(r-r_{c}\right)^{2}-c^{2}\right)\left(m^{2}-n^{2}\right)\left(r+r_{c}\right)\right]}{c\left[\left(\left(r+r_{c}\right)^{2}-c^{2}\right)-(m-n)^{2}\left(\left(r-r_{c}\right)^{2}-c^{2}\right)\right]} d \varphi .
$$

\subsection{Relation to the supersymmetric case}

The metric (3.46) is in the form used in writing the supersymmetric solutions, so we can make a detailed comparison to the supersymmetric case. If we set $m=n+1$, this metric should reduce to the familiar two-center supersymmetric solution of [14, 15, 16]. Indeed, for $m=n+1$, we will have $\gamma=1, V=4\left[(n+1) r-n r_{c}\right]^{2}$,

$$
H=\frac{n+1}{r_{c}}-\frac{n}{r}
$$

and

$$
\sigma=d \tau+\left[(n+1) \cos \theta_{c}-n \cos \theta\right] d \varphi,
$$

so the base space (3.50) reduces to the usual Gibbons-Hawking base of the two-center supersymmetric solution. This base space plays an important role in the study of the supersymmetric solutions.

Compared to the supersymmetric cases, the obvious novelty in these solutions is that the base metric (3.50) is no longer of the Gibbons-Hawking form. Indeed, although it is still a $U(1)$ fibration over a three-dimensional base space, that space is no longer flat, or even conformally flat, and the function $H$ does not appear to satisfy a harmonic equation on this base. There is no clear sign of any linear structure in the equations satisfied by this solution which could be exploited to generate multi-center solitons as in the supersymmetric case.

In the supersymmetric case, an important insight gained from the analysis of the two-center solutions in [16] was that the signature of the base space switched where $H=0$. That is, the base space was not really a hyper-Kähler manifold, but only pseudo-hyper Kähler. There is thus a much bigger space of possibilities for the base metric. In the form (3.50), we still have that for $f<0, H<0$, and for $f>M$, $H>0$, so the base has regions of positive and negative signature. However, because $V<0$ in the intermediate region $0<f<M, H$ as defined in (3.51), and hence the $4 \mathrm{~d}$ metric (3.50), will become imaginary in this intermediate region. Just as in the supersymmetric case, the full geometry (3.46) has real components and a definite signature everywhere; this imaginary function is just an artifact of how we have chosen to decompose the metric into a base and fiber. It hence does not prevent us from using this decomposition to analyse the equations of motion, analogous to what was done for the supersymmetric case. 
This suggests that if we wanted to look for further non-supersymmetric solutions using this kind of decomposition, we would need to allow possibilities which included a region where the base metric becomes imaginary. However, the absence of any nice harmonic behaviour in this form of the solution suggests that, unlike in the supersymmetric case, this decomposition may not be particularly useful.

\section{Structure of the solution}

We now analyse the structure of the solitons in this M-theory form, using the adapted coordinates. In [1], it was shown that the type IIB solution (2.1) was completely smooth, but the dualities we have performed mix up the gauge fields and the geometry, so they can change the smoothness properties of the metric. We focus on analysing the coordinate singularities at $\rho=0$ or $x= \pm 1$. We will see that there are orbifold singularities in the M-theory form. We will also see that the adapted coordinates are convenient for understanding the structure of the solution.

It is clear from (3.18) that at $x=1$, the circle along $\partial_{\phi}$ shrinks smoothly to zero size, while at $x=-1$, the circle along $\partial_{\psi}$ shrinks smoothly to zero size. The component of the gauge field along the circle which shrinks goes to zero, so the gauge fields are also smooth there.

At $\rho=0$, a circle which is a combination of these two shrinks; to analyse this region, it is convenient to introduce a different set of angular coordinates. We define

$$
\phi^{\prime}=-l \phi-k \psi, \quad \psi^{\prime}=n \phi+m \psi
$$

where $k, l$ are integers such that $k n-m l=1$. Since we assume $m, n$ are coprime, we can always find such integers 2 We introduce them so that the identifications $\phi \sim \phi+2 \pi, \psi \sim \psi+2 \pi$ are equivalent to $\phi^{\prime} \sim \phi^{\prime}+2 \pi, \psi^{\prime} \sim \psi^{\prime}+2 \pi$. In these coordinates,

$$
k=\frac{\sqrt{Q_{1} Q_{2} Q_{3}}}{\sqrt{m n} c V}\left[m n \rho^{2} d \phi^{\prime}+\frac{1}{4}\left[-\left(m^{2}-n^{2}\right)\left(1-x^{2}\right)-2 \rho^{2} x+2(k n+m l) \rho^{2}\right] d \psi^{\prime}\right],
$$

and

$$
\begin{aligned}
d \bar{s}_{4}^{2} & =\frac{d \rho^{2}}{\left(\rho^{2}+1\right)}+\frac{1}{4} \frac{d x^{2}}{\left(1-x^{2}\right)}+\rho^{2} d \phi^{\prime 2}+\frac{\rho^{4}}{V}\left[-\left(m^{2}-n^{2}\right) x-\left(m^{2}+n^{2}\right)+2\left(\rho^{2}+1\right)\right] d \phi^{\prime 2} \\
& +\frac{\rho^{2}}{V}\left[-\left(m^{2}-n^{2}\right)(k m-n l)\left(1-x^{2}\right)-2(k m-n l) x\left(\rho^{2}+1\right)+2(k m+n l)\left(\rho^{2}+1\right)\right] d \phi^{\prime} d \psi^{\prime} \\
& +\frac{\left(1-x^{2}\right)}{V} d \psi^{\prime 2} \\
& +\frac{\rho^{2}}{V}\left[-\left(k^{2}-l^{2}\right)\left(m^{2}-n^{2}\right)\left(1-x^{2}\right)-2\left(k^{2}-l^{2}\right) x\left(\rho^{2}+1\right)+2\left(k^{2}+l^{2}\right)\left(\rho^{2}+1\right)\right] d \psi^{\prime 2} .
\end{aligned}
$$

\footnotetext{
${ }^{2}$ If $m, n$ are not coprime, we could write $m=a \bar{m}, n=a \bar{n}$, with $\bar{m}, \bar{n}$ coprime, and proceed as above, with $k \bar{n}-l \bar{m}=1$. In this case, there will be a $\mathbb{Z}_{a}$ orbifold singularity at $\rho=0$.
} 
Thus at $\rho=0$, the circle along $\partial_{\phi^{\prime}}$ shrinks smoothly to zero size.

The component of the gauge field along $\partial_{\phi^{\prime}}$ is

$$
\left(A_{i}\right)_{\phi^{\prime}}=-\sqrt{\frac{Q_{j} Q_{k}}{Q_{i}}} \sqrt{m n}\left(1-\frac{4 c \rho^{2}}{\tilde{H}_{i}}\right) .
$$

As $\rho \rightarrow 0$, it goes to a constant, which gives a non-zero holonomy around the shrinking circle, $\oint_{\phi^{\prime}} A_{i}=-2 \pi \sqrt{\frac{Q_{j} Q_{k}}{Q_{i}}} \sqrt{m n}$. Can this be removed by a large gauge transformation? In the type IIB picture, the gauge field associated with $q_{p}$ comes from a Kaluza-Klein reduction, so we know that $A_{p} \rightarrow A_{p}+R d \phi^{\prime}$ is an allowed large gauge transformation. Using (2.5-2.7), we can show $R=\sqrt{\frac{q_{1} q_{5}}{q_{p}}} \sqrt{m n}$. Regarding each of the gauge fields in turn as arising as the Kaluza-Klein gauge field in a distinct reduction from type IIB, we can argue that

$$
A_{i} \rightarrow A_{i}+\sqrt{\frac{Q_{j} Q_{k}}{Q_{i}}} \sqrt{m n} d \phi^{\prime}
$$

are indeed allowed large gauge transformations. That is, the gauge group is a circle of size $2 \pi \sqrt{\frac{Q_{j} Q_{k}}{Q_{i}}} \sqrt{m n}$, and the holonomy around any cycle takes values in this circle, so any holonomy that is an integer multiple of the size of the circle is actually equivalent to zero holonomy. Thus, the gauge fields have zero holonomy up to gauge transformations, and the gauge fields are also smooth at $\rho=0$.

We should finally consider what happens at $\rho=0, x= \pm 1$, where two circles shrink simultaneously. There will be orbifold singularities at these points.

\subsection{Orbifold singularities}

The most interesting feature of the non-supersymmetric solitons in this five-dimensional picture is the way in which they differ from the supersymmetric case in their local structure near the points $\rho=0, x= \pm 1$. As in the supersymmetric case, there are orbifold singularities at these points. However, in the present case these are nonsupersymmetric orbifolds. Hence, the supersymmetry is broken not just in the full asymptotically flat solution, but even by the local solution describing the geometry near the singular points. This is somewhat surprising, as supersymmetry is not broken locally in the type IIB solution. In this section, we will explore the local structure, showing that the supersymmetry is broken locally, and explain the relation to the type IIB solution. We conclude from this local breaking of the supersymmetry that the picture of the smooth supersymmetric solutions as made up of $1 / 2$ BPS 'atoms' [24] does not extend to the present case.

Let us consider the point $\rho=0, x=1$, and study it in the $r, \theta$ coordinates introduced in section 3.2, where it corresponds to $r=0$. Near $r=0, V \approx 4 n^{2}$, $\tilde{H}_{i} \approx 2 c\left[-\left(m^{2}-n^{2}\right)+1+E_{i}\right]$ is a constant, and $k \approx 0$, so the full geometry decomposes as a flat $\mathbb{R}^{1,6}$ cross the four-dimensional base space (3.50). In the base space, $r_{c} \approx$ 
$c+r \cos \theta$,

$$
\gamma \approx \frac{1}{2}\left[(1+\cos \theta)+(m-n)^{2}(1-\cos \theta)\right]
$$

and

$$
H \gamma=\frac{2 c \sqrt{V}}{4 r r_{c}} \approx \frac{n}{r}
$$

Finally, the fiber is

$$
\sigma \approx d \tau+\frac{1}{2 \gamma}\left[(1+\cos \theta)+\left(m^{2}-n^{2}\right)(1-\cos \theta)\right] d \varphi .
$$

We introduce a new radial coordinate $\tilde{r}=\sqrt{r}$, so the base space can be written, up to an irrelevant constant scale factor, as

$$
d s_{4}^{2}=d \tilde{r}^{2}+\tilde{r}^{2}\left(\frac{d \theta^{2}}{4}+\frac{1}{4 \gamma} \sin ^{2} \theta d \varphi^{2}+\frac{\gamma}{4 n^{2}} \sigma^{2}\right) .
$$

The geometry looks locally like a cone over

$$
d \Sigma^{2}=\frac{d \theta^{2}}{4}+\frac{1}{4 \gamma} \sin ^{2} \theta d \varphi^{2}+\frac{\gamma}{4 n^{2}} \sigma^{2} .
$$

This is an orbifold of $S^{3}$. If we start from $S^{3}$ in the standard form

$$
d \Sigma^{2}=\frac{d \theta^{2}}{4}+\frac{1}{4} \sin ^{2} \theta d \varphi^{\prime 2}+\frac{1}{4}\left(d \tau^{\prime}-(1-\cos \theta) d \varphi^{\prime}\right)^{2},
$$

we can obtain (4.10) by the transformation

$$
\begin{aligned}
\tau^{\prime} & =\frac{1}{n}(\tau+\varphi), \\
\varphi^{\prime} & =-\frac{(m-n-1)}{2 n} \tau-\frac{(m+n-1)}{2 n} \varphi .
\end{aligned}
$$

The periodicities (3.23) of $\tau$ and $\varphi$ in our solution then imply that $\tau^{\prime}$ and $\varphi^{\prime}$ are identified under $\varphi^{\prime} \sim \varphi^{\prime}-2 \pi$ and $\left(\tau^{\prime}, \varphi^{\prime}\right) \sim\left(\tau^{\prime}+4 \pi / n, \varphi^{\prime}-(m-n-1) 2 \pi / n\right)$. This defines a freely-acting $\mathbb{Z}_{n}$ quotient of $S^{3}$, which is referred to in the mathematical literature as a lens space, denoted $L(n, m)$ [28, 29] $]^{3}$ In the supersymmetric case $m=n+1$, this is $L(n, 1)$, and the second identification becomes $\tau^{\prime} \sim \tau^{\prime}+4 \pi / n$, so the quotient just acts on the canonical fiber of the $S^{3}$. This is no longer true in the non-supersymmetric cases.

There is thus a $\mathbb{Z}_{n}$ orbifold singularity at $r=0$. We can make this manifest by defining complex coordinates

$$
u_{1}=\tilde{r} \sin \frac{\theta}{2} e^{i \tau^{\prime} / 2}=\tilde{r} \sin \frac{\theta}{2} e^{i(\tau+\varphi) / 2 n},
$$

\footnotetext{
${ }^{3}$ Note that the lens spaces $L(n, m)$ and $L\left(n, m^{\prime}\right)$ are homeomorphic for $m= \pm m^{\prime} \bmod n$ or $m m^{\prime}= \pm 1 \bmod n$.
} 


$$
u_{2}=\tilde{r} \cos \frac{\theta}{2} e^{i\left(\tau^{\prime}-2 \varphi^{\prime}\right) / 2}=\tilde{r} \cos \frac{\theta}{2} e^{i[(m-n) \tau+(m+n) \varphi] / 2 n},
$$

in terms of which the local geometry is simply flat $\mathbb{C}^{2}$, and the identification $\tau \sim \tau+4 \pi$ acts as

$$
u_{1} \sim u_{1} e^{2 \pi i / n}, \quad u_{2} \sim u_{2} e^{2 \pi i(m-n) / n} .
$$

The geometry is thus a non-supersymmetric $\mathbb{C}^{2} / \mathbb{Z}_{n}$ orbifold of the kind discussed for example in [30, 31]. The supersymmetry is broken except in the special case $m=n+1$; the identifications (4.16) do not preserve any Killing spinors. If $m=n+1$, it is the usual supersymmetric $\mathbb{C}^{2} / \mathbb{Z}_{n}$ orbifold (note the fermions are periodic under $\tau \sim \tau+4 \pi$ in the full geometry, so we have the supersymmetry-preserving choice of spin structure on the orbifold).

However, this is not the end of the story; we also need to consider the gauge fields. Near $r=0$,

$$
A_{i}=\frac{Q_{i}}{\tilde{H}_{i}} d t+\sqrt{\frac{Q_{j} Q_{k}}{Q_{i}}} \frac{\sqrt{m n}}{2} d \tau^{\prime} .
$$

Thus, the holonomy of the gauge field around the orbifold circle is

$$
\oint_{\tau^{\prime}} A_{i}=2 \pi \sqrt{\frac{Q_{j} Q_{k}}{Q_{i}}} \sqrt{m n} \frac{1}{n} .
$$

This is a fractional holonomy; it cannot be removed by a large gauge transformation, which can only shift the holonomy by an integer multiple of $2 \pi \sqrt{\frac{Q_{j} Q_{k}}{Q_{i}}} \sqrt{m n}$, as argued in the previous section 4

The presence of this holonomy makes this orbifold qualitatively different from the one considered in [30, even though the geometry is the same. It makes the total space of the gauge bundle regular at $r=0$. Although the direction the orbifold acts on in the base shrinks to zero size at $r=0$, the orbifold also involves a shift along the fiber given by (4.18), so there is a free action on the total space. Thus, the local orbifold singularity will be "frozen"; as in 32, the presence of this non-trivial holonomy prevents us from resolving the singularity by deforming it to a smooth ALE space with trivial fundamental group. Note that the singularity is frozen even in the supersymmetric case $m=n+1$. This freezing of the singularity also suggests that there is no tachyon in the winding sectors in this non-supersymmetric orbifold as there is no natural endpoint for the condensation of such a tachyon. Indeed, we will see below that there is no tachyon, by considering the relation to the type IIB description.

The fact that the total space of the gauge bundle is smooth also explains how this non-supersymmetric orbifold can arise from a smooth geometry in six dimensions. In the Kaluza-Klein reduction to five dimensions, the fiber always has finite size, so we might be surprised that there is a singularity in the base, but the fractional holonomy

\footnotetext{
${ }^{4}$ Note that the gauge field is however well-behaved in the covering space $S^{3}$, as we would in general expect. That is, this gauge field can be thought of locally as arising from the orbifolding of a well-behaved gauge connection on the covering space.
} 
explains how this arises: even though the total space is smooth and the fiber is not degenerating, the connection is not well-behaved at this point.

Since the type IIB solution is smooth, it is also supersymmetric locally, in a neighbourhood of any point 5 but the orbifold we obtained locally at $r=0$ is nonsupersymmetric. It is useful to consider carefully what happens to the supersymmetry under the duality from the type IIB to the M-theory picture. The key step is the reduction to a five-dimensional solution from the type IIB solution (2.1) on $S_{y}^{1} \times$ $T^{4}$. Recall that in the type IIB solution, there is a circle which shrinks smoothly to zero size at $\bar{r}=r_{+}$. The solution thus has a unique spin structure, which is antiperiodic around this circle, and also around each of the two contractible $S^{1} \mathrm{~s}$ in $S^{3}$ (parametrized by the coordinates $\phi$ and $\psi$ above). Imposing the latter two conditions, the Killing spinor must be of the general form

$$
\epsilon=e^{i \epsilon_{1} \alpha \frac{y}{2 R}} e^{i \epsilon_{2} \frac{\phi}{2}+i \epsilon_{3} \frac{\psi}{2}} f(\bar{r}, \bar{\theta}) \epsilon_{0}
$$

for some real $\alpha$ and constant spinor $\epsilon_{0}$, with three independent sign choices $\epsilon_{1}, \epsilon_{2}, \epsilon_{3}=$ \pm 1 . To impose the antiperiodicity on the degenerating circle, note that going once around the circle which shrinks to zero at $\bar{r}=r_{+}$corresponds to going once around the $y$ circle, $-m$ times around the $\phi$ circle, and $n$ times around the $\psi$ circle. Thus, we must have $\alpha=1+\epsilon_{1} \epsilon_{2} m-\epsilon_{1} \epsilon_{3} n$ to produce the correct antiperiodic behaviour around this circle. That is, the Killing spinor will be

$$
\epsilon=e^{i\left(\epsilon_{1}+\epsilon_{2} m-\epsilon_{3} n\right) \frac{y}{2 R}} e^{i \epsilon_{2} \frac{\phi}{2}+i \epsilon_{3} \frac{\psi}{2}} f(\bar{r}, \bar{\theta}) \epsilon_{0}=e^{i \epsilon_{1} \frac{y}{2 R}} e^{i \epsilon_{2}\left(m \frac{y}{2 R}+\frac{\phi}{2}\right)} e^{-i \epsilon_{3}\left(n \frac{y}{2 R}-\frac{\psi}{2}\right)} f(\bar{r}, \bar{\theta}) \epsilon_{0} .
$$

We get a five-dimensional solution by reducing on the $y$ circle. In the case $m=n+1$, where the whole solution is supersymmetric, the Killing spinor (4.20) with $\epsilon_{2}=\epsilon_{3}=$ $-\epsilon_{1}$ is constant around the $y$ circle. For the non-supersymmetric solutions, the Killing spinor (4.20) will not be constant around the $y$ circle for any choice of signs. As a result, it does not give rise to a Killing spinor in the lower-dimensional theory. To see this, note that we obtain the five-dimensional gravitino from the constant mode of the six-dimensional gravitino 6 Thus, we cannot obtain a supersymmetry in the fivedimensional solution from the supersymmetry in the six-dimensional solution: the Killing spinor which provides the supersymmetry parameter for the six-dimensional solution descends to a spinor field on the five-dimensional solution which is charged under the Kaluza-Klein gauge field, so it cannot be the parameter for a variation of the gravitino, which is not charged under the Kaluza-Klein gauge field.

That is, although the local six-dimensional geometry has a supersymmetry even for non-supersymmetric solitons, this supersymmetry is not visible in the five-dimensional solution. This is an example of the phenomenon of "supersymmetry without supersymmetry" 33. It may seem surprising that there is such a connection between

\footnotetext{
${ }^{5}$ And if we take one charge small, the type IIB solution will have a near-core region which is approximately $\mathrm{AdS}_{3} \times S^{3}$, so supersymmetry is actually restored in the whole near-core region.

${ }^{6}$ This is true if we work in the sector where $m+n$ is odd. If $m+n$ is even, the spin structure on the six-dimensional solution does not give rise to a spin structure on the five-dimensional solution, as we are Kaluza-Klein reducing on a circle with antiperiodic boundary conditions for the fermions.
} 
whether the local orbifold singularities in the five-dimensional solution preserve supersymmetry and whether the full type IIB solution does; but in both cases, the condition to preserve supersymmetry is that the Killing spinor is constant around the $y$ circle. 7

This relation to the type IIB solutions also implies that there is no instability of the local geometry. That is, there will not be any winding tachyon modes of the type studied in [30]. The absence of tachyons is clear from the type IIB solution: we would not expect any tachyon modes in the smooth soliton geometry, and in the cases where there is a near-core $\mathrm{AdS}_{3} \times S^{3}$ geometry, this supersymmetric solution is known to have no tachyons. Intuitively, we can understand the effect of the holonomy as requiring twisted sector strings to "stretch" along the fiber direction, making an additional contribution to their energy and lifting the tachyons up to positive masssquared.

To summarize our analysis of the M-theory form of the solitons, we have found that the five-dimensional solution is smooth except at two points, where we have non-supersymmetric orbifold singularities. Thus, the solution has a "two-center" structure, which is similar to the simplest supersymmetric solitons. However, these centers do not preserve any supersymmetry, even locally. This is one of the main lessons from our analysis: the picture of the supersymmetric solutions in [24], in which they were understood as built up of 1/2 BPS "atoms", does not extend to the non-supersymmetric solutions of [1]. Even the atoms are not supersymmetric. It would be very interesting to have some further characterization of what atoms may be possible.

\section{$5 \quad$ Reduction to four dimensions}

In this section we examine Kaluza-Klein reductions of our solution to four dimensions. Our ambition in considering these reductions was to find a description of the non-BPS atoms in terms of brane systems in IIA. Unfortunately, we have failed to achieve this, but it is perhaps instructive to explain how it goes wrong.

We would like to have a reduction which is smooth away from the centers at $\rho=0, x= \pm 1$. Considering the local geometry near $r=0$, this amounts to asking for a representation of the lens space (4.10) as an $S^{1}$ bundle over some smooth twodimensional manifold. In the supersymmetric case $m=n+1, \gamma=1$, and (4.10) is already of the desired form: the $S_{\tau}^{1}$ is fibered over $S^{2}$. However, in the general case, there is a problem. Since $\gamma(\theta=0)=1$, the two-dimensional space is still smooth at $\theta=0$, but $\gamma(\theta=\pi)=(m-n)^{2}$, so the two-dimensional space has a $\mathbb{Z}_{|m-n|}$ orbifold singularity along $\theta=\pi$. In the full four-dimensional geometry, this will form a line conical singularity connecting the two centers.

Is there some other, inequivalent way to represent the lens space as an $S^{1}$ bundle that avoids this problem? Start from the metric in the manifestly locally $S^{3}$ form

\footnotetext{
${ }^{7}$ In the full type IIB solution, this arises from the fact that in the asymptotically flat region, the only possible Killing spinors are constant around the $y$ circle.
} 
(4.11), and consider the general $S^{1}$, which is

$$
\left(\tau^{\prime}, \varphi^{\prime}\right) \sim\left(\tau^{\prime}+4 \pi \frac{e}{n}, \varphi^{\prime}-2 \pi \frac{(m-1) e}{n}+2 \pi f\right)
$$

for some coprime integers $e, f B$ We introduce coordinates $\bar{\tau}$ and $\bar{\varphi}$ through

$$
\begin{gathered}
\tau^{\prime}=\frac{e}{n} \bar{\tau}+2 \frac{g}{n} \bar{\varphi} \\
\varphi^{\prime}=\frac{1}{2}\left(-\frac{(m-1) e}{n}+f\right) \bar{\tau}+\left(-\frac{(m-1) g}{n}+h\right) \bar{\varphi}
\end{gathered}
$$

where $g, h$ are integers such that $e h-f g=1$. The $S^{1}$ cycle (5.1) is along $\partial_{\bar{\tau}}$ in these coordinates, and the identifications are $\bar{\tau} \sim \bar{\tau}+4 \pi, \bar{\varphi} \sim \bar{\varphi}+2 \pi$. Rewriting (4.11) in these coordinates, it becomes

$$
d \Sigma^{2}=\frac{d \theta^{2}}{4}+\frac{1}{4 G} \sin ^{2} \theta d \bar{\varphi}^{2}+\frac{G}{4 n^{2}} \bar{\sigma}^{2}
$$

where $\bar{\sigma}=d \bar{\tau}+F d \bar{\varphi}$ (the precise form of $F$ will not be necessary for our arguments), and

$$
G=\sin ^{2} \theta((m-1) e-n f)^{2}+\left[e+\frac{1}{2}(1-\cos \theta)((m-1) e-n f)\right]^{2} .
$$

Thus, we can make the two-dimensional metric smooth at $\theta=0$ by choosing $e= \pm 1$, and we can make it smooth at $\theta=\pi$ by choosing $m e-n f= \pm 1.9$ But we cannot satisfy both conditions simultaneously unless $m= \pm 1 \bmod n$, that is, unless we are considering the lens space $L(n, 1)$ which arises in the supersymmetric case.

The conclusion is thus that in the general case, the best we can do is to make the solution smooth on one of the two axes near $r=0$. It seems sensible to keep the solution smooth at large distances; we thus turn to considering in a little more detail the reduction along $\partial_{\tau}$, which has a $\mathbb{Z}_{|m-n|}$ conical singularity connecting the two centers. To do so, we first need to rewrite the metric in the usual Kaluza-Klein form. The metric is of the general form

$$
d s_{5}^{2}=-A(d t+\beta(d \tau+\delta d \varphi)+\omega d \varphi)^{2}+B(d \tau+\delta d \varphi)^{2}+C d s_{3}^{2},
$$

where $\delta$ was given in (3.21) and $d s_{3}^{2}$ was given in (3.22). It can be written as

$d s_{5}^{2}=\left(B-A \beta^{2}\right)\left[d \tau+\delta d \varphi-\frac{A \beta}{B-A \beta^{2}}(d t+\omega d \varphi)\right]^{2}-\frac{A B}{B-A \beta^{2}}(d t+\omega d \varphi)^{2}+C d s_{3}^{2}$.

\footnotetext{
${ }^{8}$ If $e, f$ are not coprime, the cycle is not primitive - it is an integer multiple of some other cycle, and we should consider instead the corresponding primitive cycle.

${ }^{9}$ This corresponds to a reduction of the full solution along the $\psi^{\prime}$ coordinate. We can easily see that the base metric (3.18) will be smooth at $\rho=0$ for such a reduction, but will have conical singularities along $x= \pm 1$. Locally near $\rho=0$, these two reductions are analogous to the reductions of a flat metric $d s^{2}=d r^{2}+r^{2} d \phi^{2}+R^{2}(d \tau+d \phi)^{2}$ with the identifications $\phi \sim \phi+\pi, \tau \sim \tau+2 \pi$. If we reduce this along $\tau$, we get a flat space with a $\mathbb{Z}_{2}$ orbifold. If we reduce instead along $\phi$, we get a smooth fluxbrane solution in two dimensions.
} 
If we then reduce along $\tau$, the last two terms will give the four-dimensional geometry.

Thus, the three-dimensional metric in (3.22) is the base space for the four-dimensional geometry: we repeat it here,

$$
d s_{3}^{2}=\frac{d \rho^{2}}{\rho^{2}+1}+\frac{d x^{2}}{4\left(1-x^{2}\right)}+\frac{\left(1-x^{2}\right) \rho^{2}\left(\rho^{2}+1\right)}{\left[4 \rho^{2}\left(\rho^{2}+1\right)+(m-n)^{2}\left(1-x^{2}\right)\right]} d \varphi^{2} .
$$

In the supersymmetric case $m=n+1$, the base metric (3.22) is flat. In the nonsupersymmetric case, it is not even conformally flat. We can see directly that it is smooth along the axes which extend to infinity, at $x= \pm 1$, and has a conical singularity between the two centers, along the line at $\rho=0$. Also, there are curvature singularities in this base metric at $\rho=0, x= \pm 1$. These are in addition to the curvature singularities in the full four-dimensional metric which will arise from the fact that the dilaton is diverging at these points.

To simplify the Kaluza-Klein reduction, it is better to change to the coordinate $\tau^{\prime}=\tau+\varphi$, so that the identifications are simply $\varphi \sim \varphi+2 \pi$ and $\tau^{\prime} \sim \tau^{\prime}+4 \pi$. From the Kaluza-Klein point of view, this is a gauge transformation which shifts $\delta \rightarrow \delta^{\prime}=\delta-1$.

The Kaluza-Klein gauge field coming from this reduction is

$$
A_{K K}=\delta^{\prime} d \varphi-\frac{A \beta}{B-A \beta^{2}}(d t+\omega d \varphi),
$$

where

$$
\omega=-\frac{\sqrt{Q_{1} Q_{2} Q_{3}}}{4 c \sqrt{m n}} \frac{(m-n) \rho^{2}\left(1-x^{2}\right)}{\left[4 \rho^{2}\left(\rho^{2}+1\right)+(m-n)^{2}\left(1-x^{2}\right)\right]},
$$

and after the above gauge transformation,

$$
\delta^{\prime}=\frac{\left[4 \rho^{2}\left(\rho^{2}+1\right)(x-1)+\left(m^{2}-n^{2}\right)\left(1-x^{2}\right) 2 \rho^{2}+2 n(m-n)\left(1-x^{2}\right)\right]}{\left[4 \rho^{2}\left(\rho^{2}+1\right)+(m-n)^{2}\left(1-x^{2}\right)\right]} .
$$

Since $\omega=0$ at $\rho=0$ and at $x= \pm 1$, the second factor in $A_{K K}$ will not contribute to holonomies at these points. We therefore do not write it more explicitly. The factor of $B-A \beta^{2}$, which will give the dilaton of the four-dimensional solution, is more complicated:

$$
\begin{aligned}
B-A \beta^{2}= & \frac{\left(\tilde{H}_{1} \tilde{H}_{2} \tilde{H}_{3}\right)^{1 / 3}}{4 V}\left[4 \rho^{2}\left(\rho^{2}+1\right)+(m-n)^{2}\left(1-x^{2}\right)\right] \\
& -\frac{Q_{1} Q_{2} Q_{3}}{\left(\tilde{H}_{1} \tilde{H}_{2} \tilde{H}_{3}\right)^{2 / 3} 16 V m n}\left\{2 \rho^{2}[(m+n)+x(m-n)]+(m-n)^{2}(m+n)\left(1-x^{2}\right)\right\}^{2} .
\end{aligned}
$$

After a lot of algebra, and using the constraint (3.32) that determines $c$ in terms of the $Q_{i}$, the factor of $V$ cancels, and this can be written as

$$
\begin{aligned}
B-A \beta^{2}= & \frac{1}{\left(\tilde{H}_{1} \tilde{H}_{2} \tilde{H}_{3}\right)^{2 / 3}}\left\{\frac{Q_{1} Q_{2} Q_{3}}{16 m n}\left[4 \rho^{2}+(m-n)^{2}\left(1-x^{2}\right)\right]\right. \\
& \left.+2 c^{3}\left[2 \rho^{2}+1-\left(m^{2}-n^{2}\right) x+E_{1}+E_{2}+E_{3}\right]\left[4 \rho^{2}\left(\rho^{2}+1\right)+(m-n)^{2}\left(1-x^{2}\right)\right]\right\} .
\end{aligned}
$$


This clearly vanishes at $\rho=0, x= \pm 1$. We will thus have singularities of the fourdimensional metric and dilaton at these points. It will not vanish at any other point, as $E_{i}>\left(m^{2}-n^{2}\right)-1$, so both terms are positive away from $\rho=0, x= \pm 1$.

In the supersymmetric case, we would view the singularities at $\rho=0, x= \pm 1$ as D6-branes. Here, however, an attempt to define the charge carried by these singularities is obstructed by non-trivial holonomies around the conical singularity. The Kaluza-Klein gauge field which arises in the reduction from five to four dimensions has a non-trivial holonomy around the conical line at $\rho=0$,

$$
\left.\oint_{\varphi} A_{K K}\right|_{\rho=0}=4 \pi \frac{n}{(m-n)} .
$$

Note that for $m=n+1$, this is an integer multiple of $4 \pi$, and hence gauge-equivalent to zero. This fractional holonomy in the general case implies that there is a deltafunction singularity in the field strength $F_{K K}$ along $\rho=0$, and we cannot associate separate Kaluza-Klein charges with the two singularities at $\rho=0, x= \pm 1$. Thus, we cannot interpret the singularities as due to the presence of D-branes at these points, since we cannot unambiguously define brane charges associated with the singularities 10

Similarly, for the gauge fields $A_{i}$, we can decompose

$$
A_{i}=A_{i}^{\prime}+\alpha\left[d \tau+\delta d \varphi-\frac{A \beta}{B-A \beta^{2}}(d t+\omega d \varphi)\right],
$$

and interpret $A_{i}^{\prime}$ as the four-dimensional gauge field 11 We find that $A_{i \varphi}^{\prime}=0$ at $x= \pm 1$. Once again, $\omega$ vanishes along $\rho^{2}=0$, so near the conical line singularity,

$$
A_{i \varphi}^{\prime} \approx A_{i \varphi}-\delta A_{i \tau}
$$

Hence at $\rho=0$ we find

$$
\left.\oint_{\varphi} A_{i}^{\prime}\right|_{\rho=0}=-2 \pi \sqrt{\frac{Q_{j} Q_{k}}{Q_{i}}} \sqrt{m n} \frac{1}{m-n},
$$

showing the same fractional holonomy for these gauge fields as well. As for the KaluzaKlein gauge field, this implies that the charges associated with the two singularities at $\rho=0, x= \pm 1$ are ambiguous.

We saw in the previous section that the M-theory solution has non-supersymmetric orbifold singularities at $\rho=0, x= \pm 1$. We had hoped that the reduction to four dimensions would clarify the interpretation of these singularities. However, the fourdimensional solution has some new features (compared to the supersymmetric case)

\footnotetext{
${ }^{10}$ Note that the total charge carried by the two singularities is still well-defined. The integral of the flux over a sphere enclosing the whole line at $\rho=0$ is $\int F_{K K}=\left.\oint_{\varphi} A_{K K}\right|_{x=1}-\left.\oint_{\varphi} A_{K K}\right|_{x=-1}=4 \pi$, so the structure carries one unit of KK monopole charge through a surface at large distance. That is, the solution is asymptotically flat in five dimensions.

${ }^{11}$ Note that $A_{i}^{\prime}$ is invariant under the gauge transformation made on $A_{K K}$, so it does not matter if we use $\tau$ and the $\delta$ of (3.21), or $\tau^{\prime}$ and the $\delta^{\prime}$ from (5.11) in carrying out this calculation. We will use the former.
} 
which make it difficult to extract a brane interpretation of these singularities. The four-dimensional solution has curvature singularities only at $\rho=0, x= \pm 1$; however, it also has a line conical singularity connecting these two curvature singularities, along $\rho=0$. This conical singularity makes it impossible to unambiguously assign charges to the two curvature singularities. Also, the curvature singularities at $\rho=0$, $x= \pm 1$ arise from a divergence of the dilaton (as in the supersymmetric case) but also from a singularity in the three-dimensional base metric at these points. It is not clear what interpretation we could give to this additional singularity from a brane construction point of view. The geometry describing a D6-brane has a flat base space, and just gets a curvature singularity from the divergence of the dilaton at the brane's position. Thus, in the non-supersymmetric case, the centers do not have a clear D-brane interpretation. It would be interesting to see whether and how this local structure is changed for non-supersymmetric solutions which are asymptotically flat in four dimensions.

\section{Acknowledgements}

We thank Henriette Elvang, Ori Ganor, and Ashish Saxena for discussions. We would also like to thank the Aspen Center for Physics where part of this work was conducted. The work of SFR was supported in part by the EPSRC and PPARC. The work of EGG was supported by the US Department of Energy under contracts DEAC03-76SF00098 and DE-FG03-91ER-40676 and by the National Science Foundation under grant PHY-00-98840.

\section{References}

[1] V. Jejjala, O. Madden, S. F. Ross, and G. Titchener, "Non-supersymmetric smooth geometries and D1-D5-P bound states," hep-th/0504181.

[2] J. M. Maldacena and L. Maoz, "De-singularization by rotation," JHEP 12 (2002) 055, hep-th/0012025.

[3] V. Balasubramanian, J. de Boer, E. Keski-Vakkuri, and S. F. Ross, "Supersymmetric conical defects: Towards a string theoretic description of black hole formation," Phys. Rev. D 64 (2001) 064011, hep-th/0011217.

[4] O. Lunin and S. D. Mathur, "Metric of the multiply wound rotating string," Nucl. Phys. B610 (2001) 49-76, hep-th/0105136.

[5] O. Lunin and S. D. Mathur, "The slowly rotating near extremal D1-D5 system as a 'hot tube'," Nucl. Phys. B615 (2001) 285-312, hep-th/0107113.

[6] O. Lunin and S. D. Mathur, "AdS/CFT duality and the black hole information paradox," Nucl. Phys. B623 (2002) 342-394, hep-th/0109154. 
[7] H. Lin, O. Lunin, and J. Maldacena, "Bubbling AdS space and 1/2 BPS geometries," JHEP 10 (2004) 025, hep-th/0409174.

[8] K. Skenderis and M. Taylor, "Fuzzball solutions and d1-d5 microstates," Phys. Rev. Lett. 98 (2007) 071601, hep-th/0609154.

[9] I. Kanitscheider, K. Skenderis, and M. Taylor, "Holographic anatomy of fuzzballs," hep-th/0611171.

[10] I. Kanitscheider, K. Skenderis, and M. Taylor, "Fuzzballs with internal excitations," arXiv:0704.0690 [hep-th].

[11] S. D. Mathur, "The fuzzball proposal for black holes: An elementary review," Fortsch. Phys. 53 (2005) 793-827, hep-th/0502050.

[12] S. D. Mathur, "The quantum structure of black holes," hep-th/0510180.

[13] S. D. Mathur, A. Saxena, and Y. K. Srivastava, "Constructing 'hair' for the three charge hole," Nucl. Phys. B680 (2004) 415-449, hep-th/0311092.

[14] S. Giusto, S. D. Mathur, and A. Saxena, "Dual geometries for a set of 3-charge microstates," Nucl. Phys. B701 (2004) 357-379, hep-th/0405017.

[15] S. Giusto, S. D. Mathur, and A. Saxena, "3-charge geometries and their CFT duals," Nucl. Phys. B710 (2005) 425-463, hep-th/0406103.

[16] S. Giusto and S. D. Mathur, "Geometry of D1-D5-P bound states," hep-th/0409067.

[17] P. Berglund, E. G. Gimon, and T. S. Levi, "Supergravity microstates for BPS black holes and black rings," hep-th/0505167.

[18] I. Bena and N. P. Warner, "Bubbling supertubes and foaming black holes," hep-th/0505166.

[19] I. Bena, C.-W. Wang, and N. P. Warner, "The foaming three-charge black hole," hep-th/0604110.

[20] J. Ford, S. Giusto, and A. Saxena, "A class of BPS time-dependent 3-charge microstates from spectral flow," hep-th/0612227.

[21] I. Bena, C.-W. Wang, and N. P. Warner, "Mergers and typical black hole microstates," JHEP 11 (2006) 042, hep-th/0608217.

[22] I. Bena and P. Kraus, "Microscopic description of black rings in AdS/CFT," JHEP 12 (2004) 070, hep-th/0408186.

[23] I. Bena and P. Kraus, "Microstates of the D1-D5-KK system," hep-th/0503053. 
[24] V. Balasubramanian, E. G. Gimon, and T. S. Levi, "Four dimensional black hole microstates: From D-branes to spacetime foam," hep-th/0606118.

[25] A. Saxena, G. Potvin, S. Giusto, and A. W. Peet, "Smooth geometries with four charges in four dimensions," JHEP 04 (2006) 010, hep-th/0509214.

[26] J. P. Gauntlett, J. B. Gutowski, C. M. Hull, S. Pakis, and H. S. Reall, "All supersymmetric solutions of minimal supergravity in five dimensions," Class. Quant. Grav. 20 (2003) 4587-4634, hep-th/0209114.

[27] S. Giusto, S. F. Ross, and A. Saxena, "Four-dimensional non-supersymmetric microstates,". in preparation.

[28] Wikipedia, "Lens space - Wikipedia, the free encyclopedia," 2007. [Online; accessed 23-April-2007].

[29] A. Hatcher, Algebraic Topology. Cambridge University Press, Cambridge, UK, 2002.

[30] A. Adams, J. Polchinski, and E. Silverstein, "Don't panic! closed string tachyons in ALE space-times," JHEP 10 (2001) 029, hep-th/0108075.

[31] J. A. Harvey, D. Kutasov, E. J. Martinec, and G. W. Moore, "Localized tachyons and RG flows," hep-th/0111154.

[32] J. de Boer et al., "Triples, fluxes, and strings," Adv. Theor. Math. Phys. 4 (2002) 995-1186, hep-th/0103170.

[33] M. J. Duff, H. Lu, and C. N. Pope, "Supersymmetry without supersymmetry," Phys. Lett. B409 (1997) 136-144, hep-th/9704186. 